\title{
Characteristics of amplified spontaneous emission of high indium content InGaN/GaN quantum wells with various silicon doping conditions
}

\author{
Yung-Chen Cheng ${ }^{1}$, En-Chiang Lin ${ }^{1}$, Shih-Wei Feng ${ }^{1}$, Hsiang-Chen Wang ${ }^{1}$, C. C. Yang ${ }^{*}, 1$, \\ Kung-Jen $\mathrm{Ma}^{2}$, Chang-Chi Pan ${ }^{3}$, and Jen-Inn Chyi ${ }^{3}$ \\ ${ }^{1}$ Graduate Institute of Electro-Optical Engineering, Department of Electrical Engineering, \\ and Gradute Institute of Electronics Enginering, National Taiwan University, 1, Roosevelt Road, Sec. 4, \\ Taipei, Taiwan \\ 2 Department of Mechanical Engineering, Chung Hua University, Hsinchu, Taiwan \\ ${ }^{3}$ Department of Electrical Engineering, National Central University, Chung-Li, Taiwan
}

Received 10 April 2003, revised 6 June 2003, accepted 23 August 2003

Published online 12 November 2003

\section{PACS 78.55.Cr, 78.67.De}

We compared the temperature dependent spectral variations of the amplified spontaneous emission (ASE) between InGaN/GaN quantum well samples of no doping, well doping, and barrier doping of silicon. The comparisons were particularly made between two series of samples with a low and a high indium content. The results show that a multi-peak ASE spectral feature and a low energy stimulated emission peak, existing at the photoluminescence shoulder, could be observed only in the high-indium-content, barrier-doped sample. Such results are supposed to originate from the formation of quantum dots of various sizes, concentrations, and shapes under the condition of barrier doping in the sample.

1 Introduction Silicon doping in InGaN/GaN quantum wells (QWs) has been widely used in fabricating high performance light emitting devices. With silicon doping, the effects of growth mode change, microstructure alternation (formation of quantum dots (QDs)-like structures), potential fluctuation reduction, strain relaxation, and piezoelectric field screening have been reported [1-5]. However, most previous studies of silicon doping effects focused on the samples emitting purple-blue photons. Usually, it is difficult to achieve a uniform InGaN alloy, particularly with high indium contents. This is because of the solid phase immiscibility and phase separation, which stem from the large lattice constant mismatch between $\mathrm{GaN}$ and InN. Because of the need of blue and green light-emitting applications, it becomes more and more important to study high indium InGaN compounds with silicon doping. In this paper, we report the characteristics of amplified spontaneous emission (ASE), photoluminescence (PL), and photoluminescence excitation (PLE) of high indium (in the green range) InGaN/GaN QWs. Also, the results of relatively low-indium-content QWs were compared to show the effects of indium content variation.

2 Sample structures and experimental procedures Two series of samples, with high and low indium contents in InGaN/GaN QWs, with different doping conditions were prepared with MOCVD growth on sapphire (0001) substrate. They all consisted of five QW periods with $2.5 \mathrm{~nm}$ in well width and $7.5 \mathrm{~nm}$ in barrier width on top of the non-doped GaN buffer layers with thickness $1.52 \mu \mathrm{m}$. The growth temperatures were 1100 and $800{ }^{\circ} \mathrm{C}$ for the GaN barriers and InGaN wells, respectively. The doping concentration of silicon was $5 \times 10^{18} \mathrm{~cm}^{-3}$, either in barriers or wells. For comparison, non-doped QW samples

" Corresponding author: e-mail: ccy@cc.ee.ntu.edu.tw, Phone: 886-2-23657624, Fax: 886-2-23652637 
were also prepared. The low and high indium samples with non-doped, well-doped and barrier-doped conditions were denoted by LU, LW, LB, HU, HW, and HB, respectively. Continuous-wave (cw) PL measurements were carried out with the $325 \mathrm{~nm}$ line of a $30 \mathrm{~mW}$ He-Cd laser. The samples were placed in a cryostat for temperature-dependent measurements ranging from 10 to $300 \mathrm{~K}$. PLE experiments were conducted using a quasi-monochromatic excitation light source from a xenon lamp dispersed by a $0.5 \mathrm{~m}$ momochromator. The emission wavelength of PLE experiments was set at the peak energy of the cw PL spectrum. The excitation length of the amplified spontaneous emission was $2.5 \mathrm{~mm}$ with the fourthharmonic $(266 \mathrm{~nm})$ of a Q-switch Nd:YAG laser $(100 \mathrm{~Hz})$ as excitation source. The excitation peak intensity was about $5 \mathrm{MW} / \mathrm{cm}^{2}$.

3 Experimental results Figure 1a shows PL spectral peak positions as functions of temperature of high-indium-content samples. A significant blue shift with barrier doping in sample HB, compared to sample HU, can be clearly seen. The PL peak position variations of samples HU (non-doped) and HW (well-doped) are close below $100 \mathrm{~K}$. However, the red shift above $100 \mathrm{~K}$ in sample HW shows the effect of well doping. The integrated PL intensities as functions of temperature are shown in Fig. 1b. The trends of significant enhancement and small change in photon emission efficiency of the barrier-doped and well-doped samples, respectively, when compared with that of the non-doped sample, are consistent with the results of low-indium samples. Figure 2 shows the temperature-dependent ASE spectra of sample HU. In this figure, the thick solid curves at the lower-left and upper-left corners show the PL spectra at room temperature and $10 \mathrm{~K}$, respectively. For temperature above $220 \mathrm{~K}$, the ASE spectra show clear Fabry-Perot resonance modes with the period corresponding to the thickness of the epi-layer of the sample. The sharp peaks at $2.33 \mathrm{eV}$ in the ASE spectra correspond to the $532 \mathrm{~nm}$ line or the second-order diffraction of the $266 \mathrm{~nm}$ line from the pumping laser. Below $220 \mathrm{~K}$, the two emission peaks could be identified as the PL peak (the lower-energy one) and the stimulated emission peak (the higher-energy one). The PL peak position of this sample is almost independent of temperature; however, the stimulated emission peak shows an S-shape temperature variation. The S-shape behavior is a result of either the carrier localization of cluster structures or the quantum-confined Stark effect. Which factor dominates depends on the sample conditions. For sample HW, the existence of two peaks and the temperaturedependent variation of the stimulated emission peak position are similar to those of the non-doped sample (HU) as shown in Fig. 3.

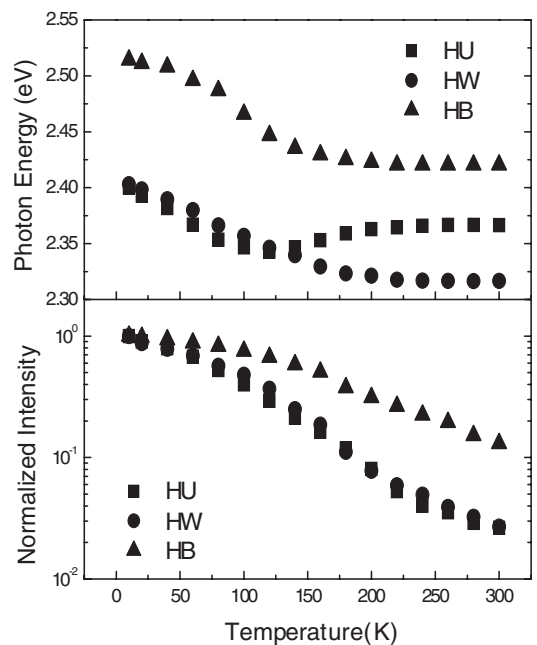

Fig. 1 Temperature-dependence PL peak energies a) and normalized integrated intensities b) of high-indium-content samples with non-doped, well-doped, and barrier conditions.

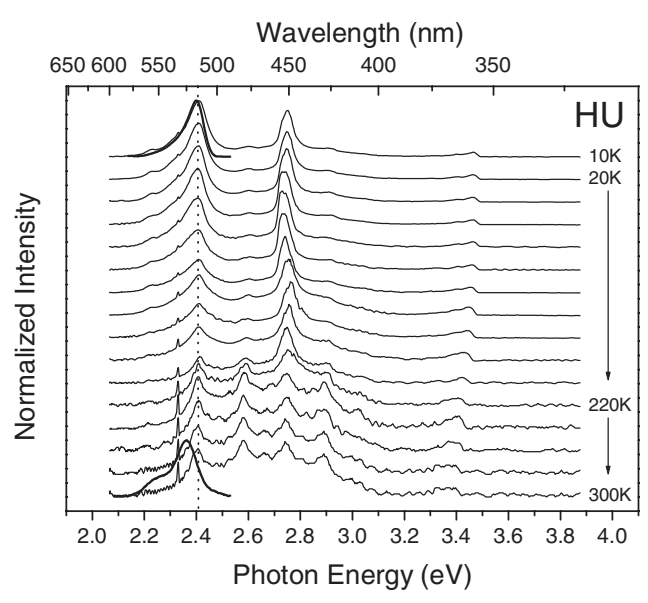

Fig. 2 ASE spectra (solid lines), $10 \mathrm{~K} \mathrm{PL}$, and $300 \mathrm{~K}$ PL spectra (thick solid lines) as functions of temperature for the non-doped high-indium-content sample (HU). 


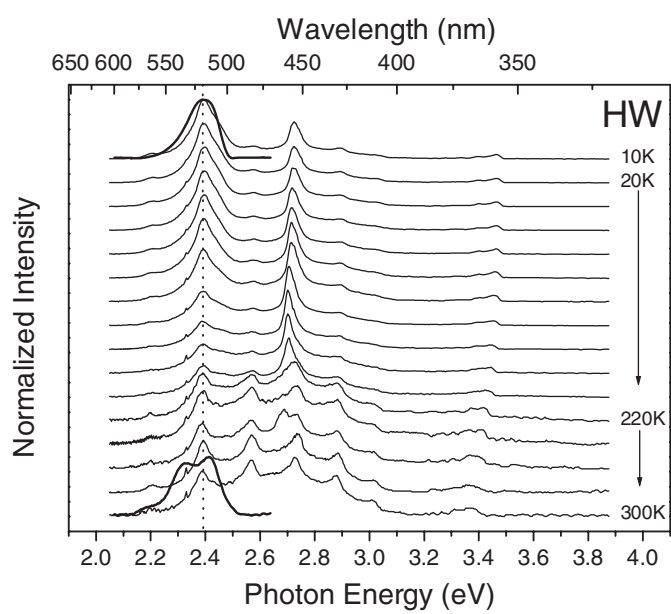

Fig. 3 ASE spectra (solid lines), $10 \mathrm{~K}$ PL, and $300 \mathrm{~K}$ PL spectra (thick solid lines) as functions of temperature for the well-doped high-indium-content sample (HW).

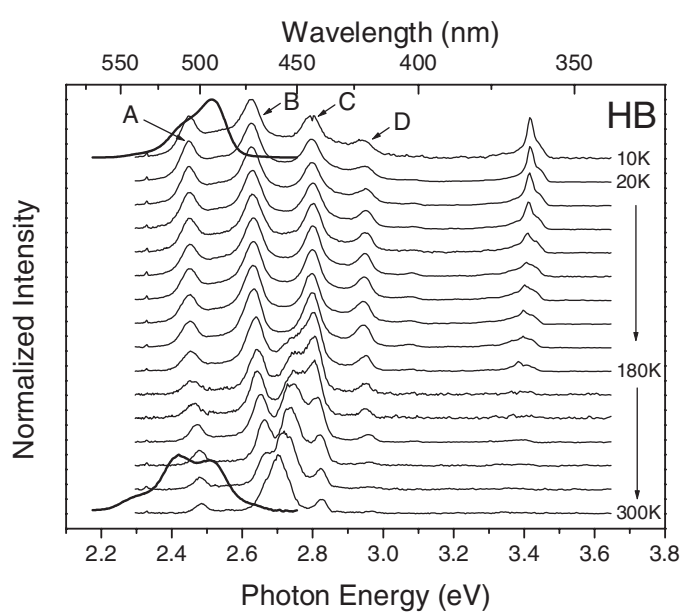

Fig. 4 ASE spectra (solid lines), $10 \mathrm{~K}$ PL, and $300 \mathrm{~K}$ PL spectra (thick solid lines) as functions of temperature for the barrier-doped high-indium-content sample (HB).

Nevertheless, as shown in Fig. 4, quite different behaviors of the temperature-dependent ASE spectrum in the barrier-doped sample (HB) were observed. The four peaks of ASE spectra in this sample are denoted with A, B, C, and D. As temperature increases, the separations among the four peaks become smaller. Also, peaks A and D diminish with increasing temperature. The multiple-peak feature evolves into a major peak around $2.7 \mathrm{eV}$ at room temperature. Figures 5 and 6 show the PL, ASE (the dashed curves), and PLE spectra at $10 \mathrm{~K}$ of the low-indium-content and high-indium-content samples, respectively. The Stokes shifts were enhanced with higher indium content. All the major stimulated emission peaks of the ASE spectra lie between the absorption peak and the PL peak. In the barrier-doped samples,

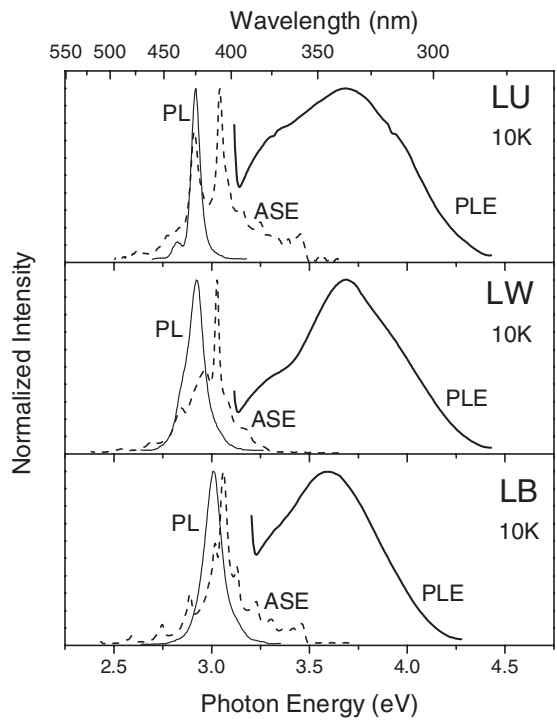

Fig. $510 \mathrm{~K}$ PL, ASE, and PLE spectra of the lowindium-content samples with undoped (LU) a), welldoped (LW) b), and barrier-doped (LB) c) conditions.

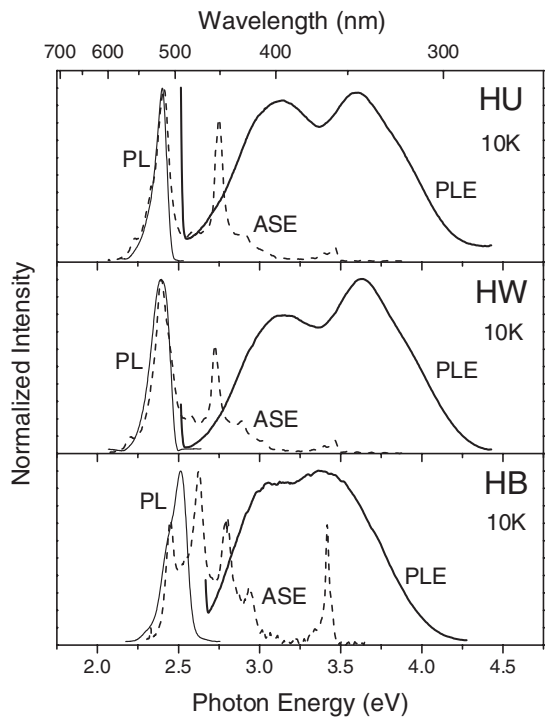

Fig. $610 \mathrm{~K} \mathrm{PL}$, ASE, and PLE spectra of the highindium-content samples with undoped (HU) a), welldoped (HW) b), and barrier-doped (HB) c) conditions. 
either high or low-indium-content, the spectral distance between the main peak of ASE and the PL peak are closer, when compared with those of the well-doped and non-doped samples. Only the high-indiumcontent, barrier-doped sample shows the multi-peak feature of stimulated emission.

4 Discussion The multiple peaks of ASE spectra of high-indium-content InGaN/GaN QWs have been observed and interpreted as various interband transitions between different quantized levels in the quantum well [6]. However, in our results only sample HB of barrier doping shows the multi-peak feature. The ASE spectra of the non-doped and well-doped samples of high-indium content show only two peaks. In our previous studies [7], it was found that silicon doping in InGaN/GaN QWs could strongly affect the microstructures. In particular, quantum-dot-like structures could be formed. Such a phenomenon was especially clear in barrier-doped samples. The origin of the multiple-peak ASE feature is attributed to the formation of indium-rich clusters of quantum nature. Such clusters have broad distributions in size, shape, indium composition, and hence a broad distribution of the photon emission spectrum. Therefore, when a plenty of carriers are generated, a quite broad gain spectrum is formed. The observed multiple ASE peaks correspond to the Fabry-Perot resonance wavelengths within the gain spectrum. Because of the edge-emitting ASE nature, such a resonance wavelength corresponds to a wave-guiding mode with the guiding layer formed with the multiple quantum well layers plus the GaN buffer layer. As temperature increases, the gain spectrum of sample HB under strong excitation becomes more centralized. In other words, the gain spectrum is effectively narrowed. Hence, we can observe the evolution of a strong ASE peak near $2.7 \mathrm{eV}$ at room temperature and the diminishing trend of peaks A and D. On the other hand, in sample HU and HW, it seems that the trends are different. Here, as temperature increases, the gain spectrum becomes broadened and a multiple-peak feature of ASE becomes visible. Such different trends reflect the differences in material microstructures. A similar feature was interpreted as the contribution of silicon impurity states [5]. However, because in our case this feature was clearly observed only in the high-indium-content, barrier-doped sample (HB), the contribution of impurity states does not seem possible.

5 Conclusions In summary, we have compared the temperature dependent ASE spectral variations between the InGaN/GaN QW samples of no doping, well doping, and barrier doping of silicon. The comparisons were particularly made between two series of the samples with low and high indium contents. It was found that a multi-peak ASE spectral feature and low energy stimulated emission peak, existing at the PL shoulder, could be observed only in the high-indium-content, barrier-doped sample (HB). Such results were supposed to originate from the formation of quantum dots of various sizes, concentrations, and shapes under the condition of barrier doping.

Acknowledgements This research was supported by National Science Council, The Republic of China, under the grants of NSC 91-2215-E-002-030 and NSC 91-2215-E-002-034, and by US Air Force under the contract AOARD02-4052.

\section{References}

[1] M. S. Minsky, S. Chichibu, S. B. Fleischer, A. C. Abare, J. E. Bowers, E. L. Hu, S. Keller, U. K. Mishra, and S. P. DenBaar, Jpn. J. Appl. Phys. 37, L1362 (1998).

[2] T. Deguchi, A. Shikanai, K. Torii, T. Sota, S. Chichibu, and S. Nakamura, Appl. Phys. Lett. 72, 3329 (1998).

[3] J. Dalfors, J. P. Bergman, P. O. Holtz, B. E. Sernelius, B. Monemar, H. Amano, and I. Akasaki, Appl. Phys. Lett. 74, 3299 (1999).

[4] C. K. Choi, Y. H. Kwon, B. D. Little, G. H. Gainer, J. J. Song, Y. C. Chang, S. Keller, U. K. Mishra, and S. P. DenBaars, Phys. Rev. B 64, 245339 (2001).

[5] T. Wang, H. Saeki, J. Bai, T. Shirahama, M. Lachab, S. Sakai, and P. Eliseev, Appl. Phys. 76, 1737 (2000).

[6] Chii-Chang Chen, Hui-Wen Chuang, Gou-Chung Chi, Chang-Cheng Chuo, and Jen-Inn Chyi, Appl. Phys. Lett. 77, 3758 (2000).

[7] Yung-Chen Cheng, Cheng-Hua Tseng, Chen Hsu, Kung-Jen Ma, Shih-Wei Feng, En-Chiang Lin, C. C. Yang, and Jen-Inn Chyi, J. Electron. Mater. 32, 375 (2003). 